\title{
\begin{tabular}{l|l|l}
\hline Site: & Patient ID number: \\
\hline
\end{tabular} \\ Heart Failure Long-Term Registry Patient Characteristics
}

\section{Inclusion criteria:}

- All outpatients with HF seen at the clinics and those admitted for acute, pre-existing or new onset HF to participating centres during the enrolment period (1 day per week).

- Chronic Heart Failure: every outpatient with chronic HF diagnosed according to the clinical judgment of the responsible cardiologist of the participating centres.

- Acute Heart Failure: patients admitted to hospital for acute HF for whom an IV therapy for HF (inotropes, vasodilators or diuretics) is needed.

\section{Exclusion criteria:}

- There are no specific exclusion criteria, with the exception of age that should be higher than 18 years.

\subsection{Basic Information}

Date of visit/hospitalisation: L______ dd/mm/yyyy

Consent :

No $\square$ Yes

If No, skip to section 6.1 CRF Completed

Type of patient ${ }^{(1)}$ : $\square$ Outpatient $\square$ Hospital

Date of birth ${ }^{(2)}$ :

L____ mm/yyyy

Sex:

Male $\square$ Female

Height:

$\mathrm{cm}$

Weight:

$\mathrm{kg}$

BMI:

Ethnic origin:

Automatic calculation

Home situation:

$\square$ Caucasian $\square$ Black

ack $\square$ Asian $\square$ Other

HF history:

$\square$ Home alone

Home with family

$\square$ Nursing home

Other

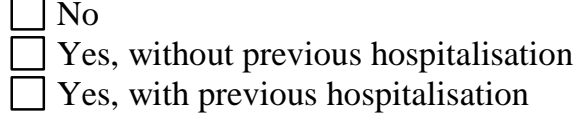

If Yes, without previous hospitalisation:
$\square<6$ months
6-12 months
$>12$ months

If Yes, with previous hospitalisation,

Date of last Hospitalisation:

Days hospitalised prior year:

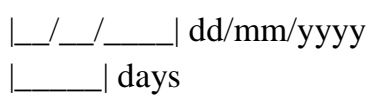

Heart rate: beats/min 
Blood pressure (Systolic/Diastolic): $\mid \_\_$__ $/$__ $\mid \mathrm{mmHg}$

Primary Etiology ${ }^{(3)}$ : $\square$ Ischemic Heart Disease documented by coronary angiography

$\square$ Ischemic Heart Disease not documented by coronary angiography

$\square$ Hypertension

$\square$ Dilated Cardiomyopathy

$\square$ Valve disease

$\square$ Tachicardia related cardiomyopathy

$\square$ HFPEF Syndrome

$\square$ Other If Other, please specify:

If Valve disease, please specify:

\begin{tabular}{|llll|}
\hline Mitral: & $\square$ No $\square$ Yes & Aortic: & $\square$ No $\square$ Yes \\
Tricuspid: & $\square$ No $\square$ Yes & Pulmonary: & $\square$ No $\square$ Yes \\
\hline
\end{tabular}

Clinical trial ${ }^{(4)}: \quad \square$ No $\square$ Yes 


\subsection{Clinical History}

\section{Risk Factors}

Smoking status ${ }^{(5)}$ :

$\begin{array}{lll}\square \text { Never } \quad \square \text { Current } & \square \text { Former } \\ \square \text { No } \quad \square \text { Permanent } & \square \text { Persistent } \square \text { Paroxysmal } \\ \square \text { No } \quad \square \text { Yes } & \square \text { Newly diagnosed } \\ \text { If Yes or Newly diagnosed, details: } & \square \text { Dietary control } \\ & \square \text { Oral medication } \\ & \square \text { Insulin } \\ & \square \text { Oral and insulin }\end{array}$

Alcohol:

Physical activities:

$\square$ Never $\square$ Former $\square$ Yes sometimes $\quad \square$ Yes daily
$\square$ None $\square$ Moderate $\square$ Intensive

\section{Previous and current condition}

\begin{tabular}{|lllll}
\hline MI/Angina: & $\square$ No $\square$ Yes & CABG: & $\square$ No $\square$ Yes \\
\hline PCI: & $\square$ No $\square$ Yes & Stroke/TIA: & $\square$ No $\square$ Yes \\
\hline Peripheral vascular disease: & $\square$ No $\square$ Yes & Valvular surgery: & $\square$ No $\square$ Yes \\
\hline Hypertension Treatment: ${ }^{(6)}$ & $\square$ No $\square$ Yes & VTE: & $\square$ No $\square$ Yes \\
\hline $\begin{array}{l}\text { COPD (Chronic obstructive } \\
\text { pulmonary disease): }\end{array}$ & $\square$ No $\square$ Yes & Chronic kidney dysfunction: ${ }^{(7)}$ & $\square$ No $\square$ Yes \\
\hline $\begin{array}{l}\text { Current malignant (cancer) } \\
\text { disease: }\end{array}$ & $\square$ No $\square$ Yes & Hepatic dysfunction: & $\square$ No $\square$ Yes \\
\hline Depression: & $\square$ No $\square$ Yes & Parkinson: & $\square$ No $\square$ Yes \\
\hline Rheumatoid arthritis: & $\square$ No $\square$ Yes & & \\
\hline
\end{tabular}

Sleep apnea: $\square$ No $\square$ Yes $\quad$ If Yes, $\begin{aligned} & \text { Sleep apnea tested: } \\ & \text { Sleep apnea treated: } \quad \square \text { No } \square \text { Yes }\end{aligned}$

Spirometry: $\square$ No $\square$ Yes $\quad$ If Yes, $\square$ Normal $\square$ Abnormal

If Abnormal, $\square$ Gold $1 \quad \square$ Gold $2 \quad \square$ Gold $3 \quad \square$ Gold 4

Forced Vital Capacity (FVC):

Forced Expiratory Volume (FEV1):

FEV/FVC:

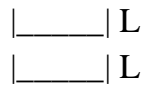

(automatic calculation)

Device therapy:

$$
\begin{aligned}
& \square \text { No } \\
& \square \text { Pacemaker } \\
& \square \text { CRT-P } \\
& \square \text { CRT-D } \\
& \square \text { ICD for primary prevention } \\
& \square \text { ICD for secondary prevention }
\end{aligned}
$$

Thyroid dysfunction: $\square$ No $\square$ Hypothyroidism $\quad \square$ Hyperthyroidism
Hepatitis $^{(8)}$ :
$\square$ No $\square$ A $\square$ B $\square$ C

Last known Ejection Fraction available:

$\square$ No $\square$ Yes

If Yes, $\mid \_$Method: $\square$ Echocardiogram $\square$ Angiography $\square$ Scintigraphy $\square$ CMR 


\section{Heart Failure Long-Term Registry \\ Outpatient visit}

(To be completed only if type of patient $=$ Outpatient

\subsection{Physical Signs}

NYHA class: $\square$ NYHA I $\square$ NYHA II $\square$ NYHA III $\square$ NYHA IV

\begin{tabular}{|ll|ll|}
\hline Pulmonary rales: & $\square$ No $\square$ Yes & S3 gallop: & $\square$ No $\square$ Yes \\
\hline JVP(>6cm): & $\square$ No $\square$ Yes & Peripheral hypoperfusion: & $\square$ No $\square$ Yes \\
\hline Pleural effusion: & $\square$ No $\square$ Yes & Cold: & $\square$ No $\square$ Yes \\
\hline Hepatomegaly: & $\square$ No $\square$ Yes & Mitral regurgitation: & $\square$ No $\square$ Yes \\
\hline Peripheral oedema: & $\square$ No $\square$ Yes & Aortic stenosis: & $\square$ No $\square$ Yes \\
\hline
\end{tabular}

\subsection{Chemistry at Outpatient Visit (most recent)}

\begin{tabular}{|c|c|c|c|c|c|}
\hline White blood cells: & | & $\begin{array}{l}\square \text { Cells/microL } \\
\square \text { Giga/L }\end{array}$ & $\begin{array}{l}\text { Total } \\
\text { cholesterol: }\end{array}$ & | & $\begin{array}{l}\square \mathrm{mg} / \mathrm{dL} \\
\square \mathrm{mmol} / \mathrm{L}\end{array}$ \\
\hline Hemoglobin: & - & $\begin{array}{l}\square \mathrm{g} / \mathrm{dL} \\
\square \mathrm{mmol} / \mathrm{L} \\
\square \mathrm{g} / \mathrm{L}\end{array}$ & Fasting glucose: & - & $\begin{array}{l}\square \mathrm{mg} / \mathrm{dL} \\
\square \mathrm{mmol} / \mathrm{L}\end{array}$ \\
\hline S-creatinine: & | & $\begin{array}{l}\square \mathrm{mg} / \mathrm{dL} \\
\square \mu \mathrm{mol} / \mathrm{L}\end{array}$ & HbA1c: & - & $\square^{\%} \%$ \\
\hline Nitrogen measured by: & $\square$ BUN & $\square$ Urea & BNP: & 1 & $\begin{array}{l}\square \mathrm{pg} / \mathrm{mL} \\
\square \mathrm{pmol} / \mathrm{L}\end{array}$ \\
\hline If $\mathrm{BUN}^{(9)}$ : & | & $\begin{array}{l}\square \mathrm{mg} / \mathrm{dL} \\
\square \mathrm{mmol} / \mathrm{L}\end{array}$ & NT-proBNP: & | & $\begin{array}{l}\square \mathrm{pg} / \mathrm{mL} \\
\square \mathrm{pmol} / \mathrm{L}\end{array}$ \\
\hline If urea: & - & \begin{tabular}{|l}
$\square \mathrm{mg} / \mathrm{dL}$ \\
$\square \mathrm{mmol} / \mathrm{L}$
\end{tabular} & Sodium: & | & $\mathrm{mEq} / \mathrm{L}$ or $\mathrm{mmol} / \mathrm{L}$ \\
\hline Uric acid: & | & $\begin{array}{l}\square \mathrm{mg} / \mathrm{dL} \\
\square \mu \mathrm{mol} / \mathrm{L}\end{array}$ & Potassium: & L & $\mathrm{mEq} / \mathrm{L}$ or $\mathrm{mmol} / \mathrm{L}$ \\
\hline Proteinuria: & $\square$ No $\square$ & & Bilirubin: & 1 & $\begin{array}{l}\square \mathrm{mg} / \mathrm{dL} \\
\square \mu \mathrm{mol} / \mathrm{L}\end{array}$ \\
\hline TSH: & | & $\mathrm{mIU} / \mathrm{L}$ & HIV infection: & $\square$ No $\square$ & Yes \\
\hline Troponin I or T: & | & $\mathrm{ng} / \mathrm{mL}$ or $\mathrm{pg} / \mathrm{mL}$ & Hs-CRP: & |__ & $\mathrm{mg} / \mathrm{L}$ \\
\hline Hs-Troponin I or T: & | & $\mathrm{ng} / \mathrm{mL}$ or $\mathrm{pg} / \mathrm{mL}$ & & & \\
\hline
\end{tabular}




\subsection{Investigations/Procedures}

(In general the most recent results at the time of the visit. If in doubt select 'Not performed')

ECG:

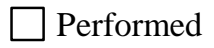

$\square$ Not performed

If performed, date:

dd/mm/yyyy

Rhythm:

Heart rate:

QRS-duration:

QT-duration:

LBBB:

LVH:

\begin{tabular}{|c|c|}
\hline ] Sinus & $\square$ Atrial \\
\hline & $\begin{array}{l}\text { beats/min } \\
\mathrm{ms}\end{array}$ \\
\hline & $\mathrm{ms}$ \\
\hline$\exists$ No & Yes \\
\hline $\begin{array}{l}\text { No } \\
\text { No }\end{array}$ & $\begin{array}{l}7 \text { Yes } \\
\text { Yes }\end{array}$ \\
\hline
\end{tabular}

Other

Pathological Q-wave: $\quad \square$ No $\square$ Yes
Chest X-ray:
$\square$ Performed
Not performed
Date: |_l___ dd/mm/yyyy

Normal?

No $\square$ Yes

If No, please specify:

\begin{tabular}{|ll|ll|}
\hline Cardiac enlargement: $\square$ No $\square$ Yes & Pulmonary congestion: $\square$ No $\square$ Yes \\
\hline Alveolar oedema: & $\square$ No $\square$ Yes & Other abnormality: $\quad \square$ No $\square$ Yes \\
\hline
\end{tabular}

If Other, please describe:

Echo-Doppler:

EF:

LVEDD:

LVH:

E/A:

Deceleration time:

LA measurement: $\square$ Performed $\square$ Not performed

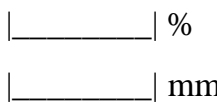

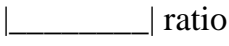

$\mathrm{ms}$
QTc-length automatic calculation

Bazett

Fridericia

$\square$ Volume $\square$ Dimension
IF Volume, LA Volume:
IF Dimension, LA Dimension:

Restrictive/pseudonormal pattern:

$\square$ No $\square$ Yes
$\square$ No $\square$ Yes
$\square$ No $\square$ Yes
$\square$ No $\square$ Yes
$\square$ No
$\square$ Yes

Mitral regurgitation moderate-severe:

Aortic stenosis moderate-severe:

Aortic regurgitation moderate-severe:

Tricuspid regurgitation moderate-severe:

Date: $\mid \_/ \ldots$ dd/mm/yyyy

Exercise test: $\square$ No $\square$ Yes $\square$ Patient cannot do test

Date: $\mid \_/ / \ldots$ dd/mm/yyy

Peak exercise, cycle ergometer:

Peak exercise, treadmill:

Peak VO2:

6 min walk test:

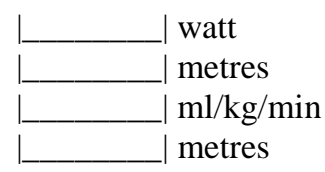

Holter Monitoring:

Performed $\square$ Not performed

Date: $\mid \_/ \ldots$ dd/mm/yyy

Mean HR:

PVC hour:

Unsustained VT:

Sustained VT:

Atrial fibrillation:

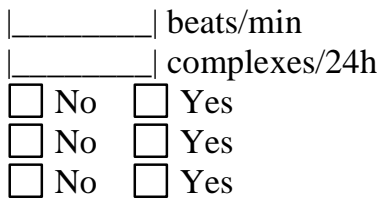

$\square$ Unknown

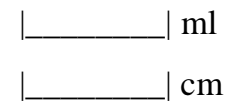

$\square$ Not evaluated 
Coronary Angiography:

Cardiac CT:

PCI/CABG:
Not performed

Performed $\square$ Not performed

Performed

Not performed

EPS (Electrophysiological Study): $\square$ Performed $\square$ Not performed Inducible sustained VT/VF ${ }^{(10)}$ : $\square$ No $\square$ Yes Inducible atrial fibrillation: $\quad \square$ No $\square$ Yes Major conduction abnormalities: $\square$ No $\square$ Yes
Date: |______ dd/mm/yyy

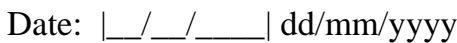

Date: |______ dd/mm/yyy

Date: |_____
Electric cardioversion: Atrial Fibrillation: VT/VF:

Right Heart Catheterization: mPAP:

Right atrial pressure: PCW:

CI:

\section{Myocardial Scintigraphy:}

Resting ischemia : Myocardial viability:

Endomyocardial Biopsy:

IAPB:

CRT implantation:

If indicated, treatment:

If not planned, reason:
Date: |______ $\mathrm{dd} / \mathrm{mm} /$ yyyy

$\square$ Performed
$\square$ No $\square$ Yes
$\square$ No $\square$ Yes
$\square$ No $\square$ Yes

Not performed

$\square$ Performed $\square$ Not performed
$\square$ No $\square$ Yes
$\square$ No $\square$ Yes

Date: |______ dd/mm/yyy

Date: |______ dd/mm/yyy

$\square$ Performed $\square$ Not performed
$\square$ No $\square$ Yes
$\square$ No $\square$ Yes

Performed $\square$ Not performed

Performed

Not performed
Date: I_____ $\mathrm{dd} / \mathrm{mm} /$ ууууу

Date: |______ dd/ d $/$ mm/yyy
Not indicated $\square$ Indicated $\square$ Already implanted

$$
\begin{aligned}
& \square \text { Not planned } \square \text { Planned } \\
& \square \text { Absence of clinical indication } \\
& \square \text { Cost issues } \\
& \square \text { Patient refusal } \\
& \square \text { Logistic issues } \\
& \square \text { Other }
\end{aligned}
$$

\section{ICD implantation:}

If indicated, treatment:

If not planned, reason: $\square$ Not indicated $\square$ Indicated $\square$ Already implanted
$\square$ Not planned $\square$ Planned
$\square$ Absence of clinical indication
$\square$ Cost issues
$\square$ Patient refusal
$\square$ Logistic issues
$\square$ Other 


\subsection{Scores}

Was prognosis evaluated using a risk score? $\quad \square$ No $\square$ Yes

$\begin{array}{ll}\text { If Yes, } \quad & \square \text { SEATLE } \\ & \square \text { CHARM } \\ & \text { GISSI-HF } \\ & \text { MAGGIC } \\ & \text { MECKI } \\ & \text { HF ACTIONS } \\ & \text { EMPHASIS } \\ & \\ \text { OTHER }\end{array}$

If Other, please specify:

References

- $\quad$ SEATLE : Wayne C; Levy et al - Circulation 2006, 113, 1424-1433

- CHARM : Stuart J. Pocock et al - European Heart Journal 2006, 27, 65-75

- GISSI-HF : Simona Barlera et al - Circulation Heart Failure 2013, 6, 31-39

- MAGGIC : Stuart J. Pocock et al - European Heart Journal 2012, 34, 1404-13

- MECKI : Piergiuseppe Agostini et al - Interventional Journal of Cardiology, 2012, 06-113

- HF ACTION: Christopher M. O’Connor et al - Circulation Heart Failure 2012, 5, 63-71

- $\quad$ EMPHASIS : Timothy J. Collier et al - European Heart Journal 2013, 34, 2823-9 


\section{Heart Failure Long-Term Registry Hospitalisation}

(To be completed only if type of patient $=$ Hospital Inpatient)

\subsection{Hospitalised patients}

First medical contact:

$\square$ Family doctor/GP
$\square$ Outpatient clinic
$\square$ Other

How did the patient arrive at the hospital?

Where was the patient first admitted?

HF status:

$\square$ Own transport $\square$ Ambulance and physician
$\square$ Ambulance and nurse $\square$ Other

$\begin{array}{ll}\square \text { Emergency room } & \square \text { Medical ward } \\ \square \text { Heart Failure facilities } & \square \text { Cardiac/Coronary ICU } \\ \square \text { Cardiac ward } & \square \text { General/Medical/Surgical ICU } \\ \square \text { Other } & \end{array}$

Ambulance and physician

Reason for hospitalisation, precipitating factors:

\begin{tabular}{|llll|}
\hline Heart Failure: & $\square$ No $\square$ Yes & ACS: & $\square$ No $\square$ Yes \\
\hline Myocardial ischemia: & $\square$ No $\square$ Yes & Non compliance behavioural drugs: \\
\hline Atrial fibrillation: & $\square$ No $\square$ Yes & Ventricular arrhythmia: & $\square$ Yes \\
\hline Infection: & $\square$ No $\square$ Yes & Uncontrolled hypertension: & $\square$ Yes \\
\hline Bradyarrhythmias: & $\square$ No $\square$ Yes & Renal dysfunction: & $\square$ Yes \\
\hline Iatrogenic: & $\square$ No $\square$ Yes & Anaemia: & $\square$ Yes \\
\hline Other: & $\square$ No $\square$ Yes & & $\square$ Yes \\
\hline
\end{tabular}

Hospital presentation, clinical profiles:

$\square$ Pulmonary oedema

$\square$ Cardiogenic shock

$\square$ Right HF
Worsening
Ambulance personnel

Nurse

Hypertensive HF

$\square$ Decompensated HF

$\square$ ACS/HF

Intropic support type:
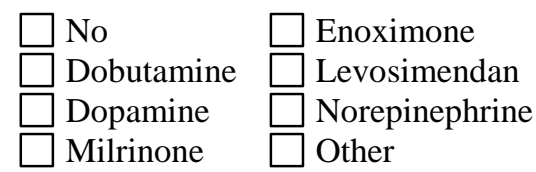

Hours of treatment:

If Other, please specify:

Nitrates IV: $\square$ No $\square$ Yes

Duration of Nitrate IV infusion: $\square<1 \mathrm{hr}$

1-3 hrs

3-6 hrs

6-12 hrs

$>12 \mathrm{hrs}$

Reason for Nitrate IV infusion terminated:

$\square$ Clinically stabilised
$\square$ Low BP
$\square$ Headache
$\square$ Tachyphylaxis
$\square$ Other

Diuretics IV:

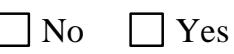




\subsection{Physical Signs}

NYHA class: $\square$ NYHA II $\square$ NYHA III $\square$ NYHA IV

\begin{tabular}{|ll|ll|}
\hline Pulmonary rales: & $\square$ No $\square$ Yes & S3 gallop: & $\square$ No $\square$ Yes \\
\hline JVP(>6cm): & $\square$ No $\square$ Yes & Peripheral hypoperfusion: & $\square$ No $\square$ Yes \\
\hline Pleural effusion: & $\square$ No $\square$ Yes & Cold: & $\square$ No $\square$ Yes \\
\hline Hepatomegaly: & $\square$ No $\square$ Yes & Mitral regurgitation: & $\square$ No $\square$ Yes \\
\hline Peripheral oedema: & $\square$ No $\square$ Yes & Aortic stenosis: & $\square$ No $\square$ Yes \\
\hline
\end{tabular}

\subsection{Chemistry at Hospital Entry}

\begin{tabular}{|c|c|c|c|c|}
\hline White blood cells: & $\mathrm{L} \_\begin{array}{l}\square \text { Cells/microL } \\
\square \text { Giga/L }\end{array}$ & $\begin{array}{l}\text { Total } \\
\text { cholesterol: }\end{array}$ & 1 & $\begin{array}{l}\square \mathrm{mg} / \mathrm{dL} \\
\square \mathrm{mmol} / \mathrm{L}\end{array}$ \\
\hline Hemoglobin: & $\mathrm{I} \_\begin{array}{l}\square \mathrm{g} / \mathrm{dL} \\
\square \mathrm{mmol} / \mathrm{L} \\
\square \mathrm{g} / \mathrm{L}\end{array}$ & Fasting glucose: & L & $\begin{array}{l}\square \mathrm{mg} / \mathrm{dL} \\
\square \mathrm{mmol} / \mathrm{L}\end{array}$ \\
\hline S-creatinine: & $\mathrm{l} \_\begin{array}{c}\square \mathrm{mg} / \mathrm{dL} \\
\square \mu \mathrm{mol} / \mathrm{L}\end{array}$ & HbA1c: & 1 & $\begin{array}{l}\square \% \\
\square \mathrm{mmol} / \mathrm{mol}\end{array}$ \\
\hline Nitrogen measured by: & $\square$ BUN $\square$ Urea & BNP: & L & $\begin{array}{l}\square \mathrm{pg} / \mathrm{mL} \\
\square \mathrm{pmol} / \mathrm{L}\end{array}$ \\
\hline If $\mathrm{BUN}^{(9)}$ : & 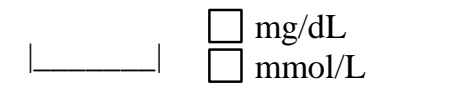 & NT-proBNP: & $\mid$ & $\begin{array}{l}\square \mathrm{pg} / \mathrm{mL} \\
\square \mathrm{pmol} / \mathrm{L}\end{array}$ \\
\hline If urea: & $-\begin{array}{c}\square \mathrm{mg} / \mathrm{dL} \\
\square \mathrm{mmol} / \mathrm{L}\end{array}$ & Sodium: & - & $\mathrm{mEq} / \mathrm{L}$ or $\mathrm{mmol} / \mathrm{L}$ \\
\hline Uric acid: & $-\begin{array}{l}\square \mathrm{mg} / \mathrm{dL} \\
\square \mu \mathrm{mol} / \mathrm{L}\end{array}$ & Potassium: & - & $\mathrm{mEq} / \mathrm{L}$ or $\mathrm{mmol} / \mathrm{L}$ \\
\hline Proteinuria: & $\square$ No $\square$ Yes & Bilirubin: & 1 & $\begin{array}{l}\square \mathrm{mg} / \mathrm{dL} \\
\square \mu \mathrm{mol} / \mathrm{L}\end{array}$ \\
\hline TSH: & |__ $\mathrm{mIU} / \mathrm{L}$ & HIV infection: & $\square$ No $\square$ & Yes \\
\hline Troponin I or T: & | $\mathrm{ng} / \mathrm{mL}$ or $\mathrm{pg} / \mathrm{mL}$ & Hs-CRP: & 1 & $\mathrm{mg} / \mathrm{L}$ \\
\hline Hs-Troponin I or T: & | $\mathrm{ng} / \mathrm{mL}$ or $\mathrm{pg} / \mathrm{mL}$ & & & \\
\hline
\end{tabular}




\section{Heart Failure Long-Term Registry Medication: (Outpatients)}

\subsection{Medications and Doses}

CV drugs:

\begin{tabular}{|c|c|c|}
\hline Drug type & Prior & During Outpatient Visit \\
\hline \multirow{23}{*}{ ACE inhibitors } & \multirow{23}{*}{$\begin{array}{l}\square \text { No } \\
\square \text { Ramipril } \\
\square \text { Enalapril } \\
\square \text { Perindopril } \\
\square \text { Lisinopril } \\
\square \text { Captopril } \\
\square \text { Fosinopril } \\
\text { Other } \\
\text { Daily dose: }|\quad| \text { mg }\end{array}$} & \multirow{8}{*}{$\begin{array}{l}\square \text { No } \\
\square \text { Ramipril } \\
\square \text { Enalapril } \\
\square \text { Perindopril } \\
\square \text { Lisinopril } \\
\square \text { Captopril } \\
\square \text { Fosinopril } \\
\square \text { Other }\end{array}$} \\
\hline & & \\
\hline & & \\
\hline & & \\
\hline & & \\
\hline & & \\
\hline & & \\
\hline & & \\
\hline & & \multirow{2}{*}{ 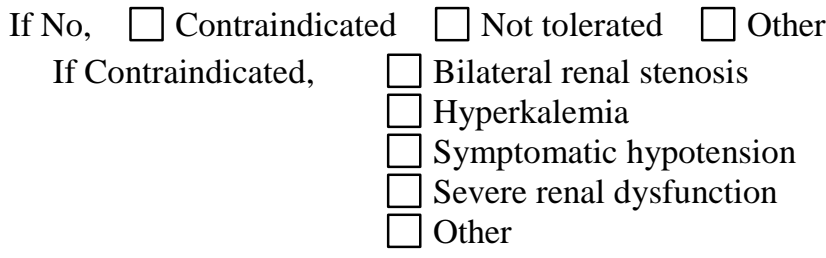 } \\
\hline & & \\
\hline & & \multirow{5}{*}{$\begin{array}{l}\square \text { Cough } \\
\square \text { Worsening renal function } \\
\square \text { Symptomatic hypotension } \\
\square \text { Hyperkalemia } \\
\square \text { Angioedema } \\
\square \text { Other }\end{array}$} \\
\hline & & \\
\hline & & \\
\hline & & \\
\hline & & \\
\hline & & \multirow{8}{*}{ 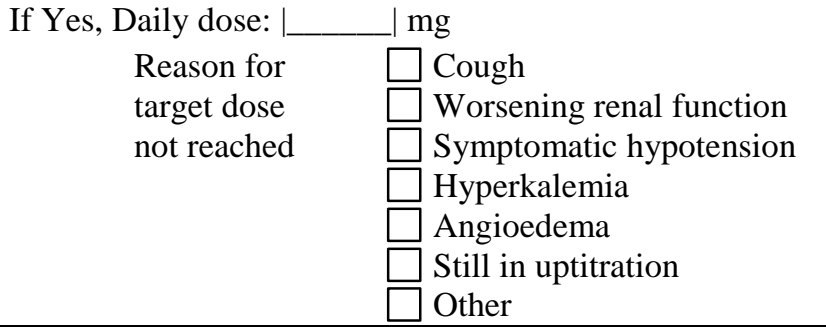 } \\
\hline & & \\
\hline & & \\
\hline & & \\
\hline & & \\
\hline & & \\
\hline & & \\
\hline & & \\
\hline \multirow{7}{*}{$\begin{array}{l}\text { Angiotensin II } \\
\text { receptor Blockers } \\
\text { (ARB) }\end{array}$} & \multirow{7}{*}{$\begin{array}{l}\square \text { No } \\
\square \text { Candesartan } \\
\square \text { Losartan } \\
\square \text { Valsartan } \\
\square \text { Other } \\
\text { Daily dose: } \mid \_ \text {mg }\end{array}$} & \multirow{5}{*}{$\begin{array}{l}\square \text { No } \\
\square \text { Candesartan } \\
\square \text { Losartan } \\
\square \text { Valsartan } \\
\square \text { Other }\end{array}$} \\
\hline & & \\
\hline & & \\
\hline & & \\
\hline & & \\
\hline & & $\begin{array}{c}\text { If No, } \square \text { Contraindicated } \square \text { Not tolerated } \square \text { Other } \\
\text { If Contraindicated, } \quad \square \text { Bilateral renal stenosis } \\
\square \text { Hyperkalemia } \\
\square \text { Symptomatic hypotension } \\
\square \text { Severe renal dysfunction } \\
\square \text { Other }\end{array}$ \\
\hline & & $\begin{array}{l}\square \text { Worsening renal function } \\
\square \text { Symptomatic hypotension } \\
\square \text { Hyperkalemia } \\
\square \text { Angioedema } \\
\square \text { Other }\end{array}$ \\
\hline
\end{tabular}




\begin{tabular}{|c|c|c|}
\hline & & 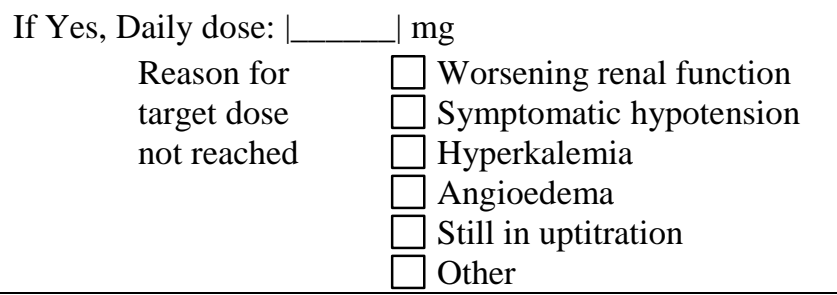 \\
\hline $\begin{array}{l}\text { Sacubitril / } \\
\text { Valsartan } \\
\text { (ARNI: } \\
\text { Angiotensin } \\
\text { Receptor } \\
\text { Neprilysin } \\
\text { Inhibitor) }\end{array}$ & $\begin{array}{l}\square \text { No } \\
\square \text { Yes } \\
\text { Daily dose: } \mid \_ \text {___ mg }\end{array}$ & $\begin{array}{l}\square \text { No } \\
\square \text { Yes } \\
\text { If No, } \square \text { Contraindicated } \square \text { Not tolerated } \square \text { Other } \\
\text { If Yes, Daily dose: }\left|\_\right| \text {mg }\end{array}$ \\
\hline \multirow[t]{3}{*}{ Beta blockers } & \multirow[t]{3}{*}{$\begin{array}{l}\square \text { No } \\
\square \text { Carvedilol } \\
\square \text { Bisoprolol } \\
\square \text { Metoprolol } \\
\square \text { Nebivolol } \\
\square \text { Other } \\
\text { Daily dose: }|\quad| \text { mg }\end{array}$} & 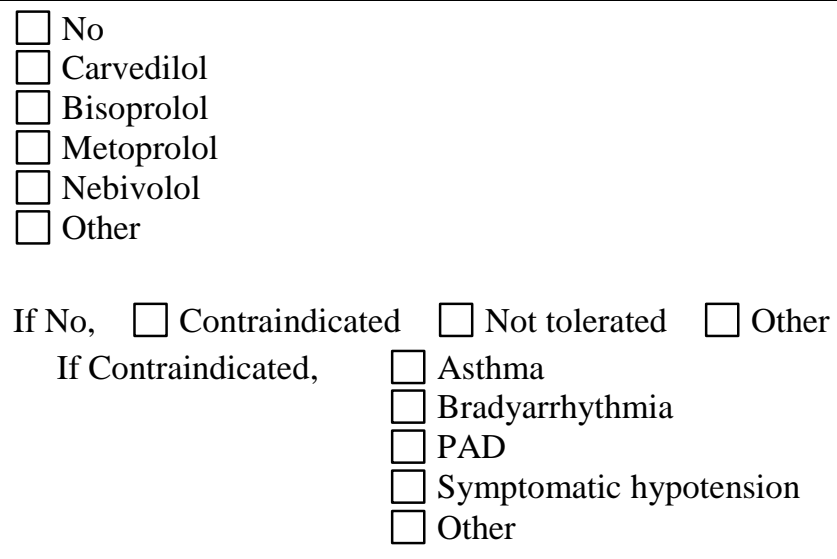 \\
\hline & & 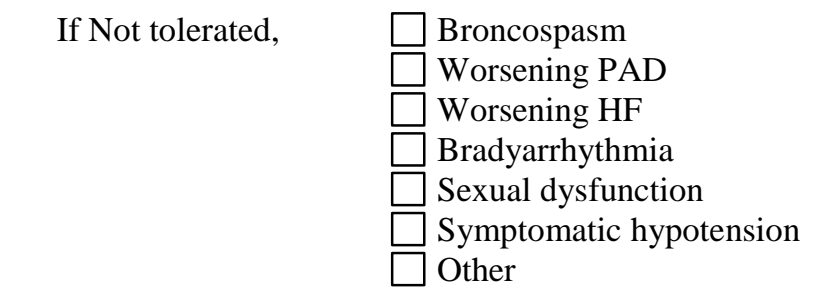 \\
\hline & & 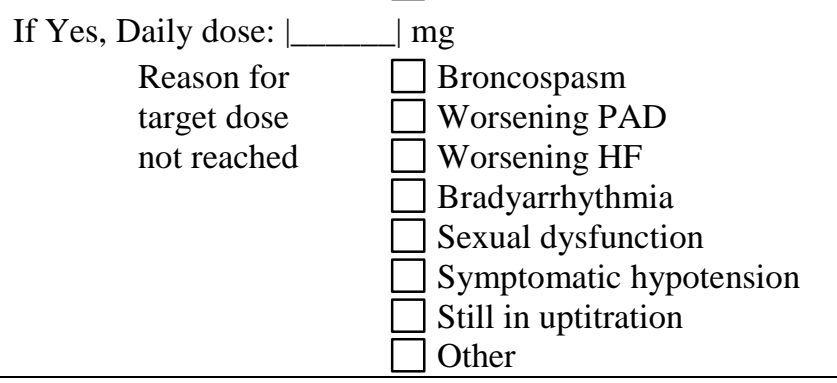 \\
\hline \multirow[t]{3}{*}{$\begin{array}{l}\text { Mineralocorticoid } \\
\text { receptor } \\
\text { antagonists } \\
\text { (Aldosterone } \\
\text { antagonists) }\end{array}$} & \multirow[t]{3}{*}{$\begin{array}{l}\square \text { No } \\
\square \text { Spironolactone } \\
\square \text { Eplerenone } \\
\square \text { Canrenone } \\
\square \text { Other } \\
\text { Daily dose: }|\quad| \mathrm{mg}\end{array}$} & \multirow{2}{*}{$\begin{array}{l}\square \text { No } \\
\square \text { Spironolactone } \\
\square \text { Eplerenone } \\
\square \text { Canrenone } \\
\square \text { Other } \\
\text { If No, } \square \text { Contraindicated } \square \text { Not tolerated } \square \text { Other } \\
\quad \text { If Contraindicated, } \quad \square \text { Hyperkalemia } \\
\end{array}$} \\
\hline & & \\
\hline & & $\begin{array}{l}\square \text { Hyperkalemia } \\
\square \text { Worsening renal function }\end{array}$ \\
\hline
\end{tabular}




\begin{tabular}{|c|c|c|}
\hline & & $\begin{aligned} & \square \text { Gynecomastie } \\
& \square \text { Other } \\
& \text { If Yes, Daily dose: } \mid \mid \mathrm{mg} \\
& \text { Reason for } \square \text { Hyperkalemia } \\
& \text { target dose } \square \text { Worsening renal function } \\
& \text { not reached } \square \text { Gynecomastie } \\
& \square \text { Still in uptitration } \\
& \square \text { Other } \\
&\end{aligned}$ \\
\hline Diuretics: Oral & $\begin{array}{l}\square \text { No } \\
\square \text { Bendrofluazide } \\
\square \text { Chlorthalidone } \\
\square \text { Hydrochlorothiazide } \\
\square \text { Furosemide } \\
\square \text { Indapamide } \\
\square \text { Torasemide } \\
\text { Bumetanide } \\
\text { Other } \\
\text { Daily dose: }|\quad| \text { mg }\end{array}$ & $\begin{array}{l}\square \text { No } \\
\square \text { Bendrofluazide } \\
\square \text { Chlorthalidone } \\
\text { Hydrochlorothiazide } \\
\square \text { Furosemide } \\
\text { Indapamide } \\
\text { Torasemide } \\
\text { Bumetanide } \\
\square \text { Other } \\
\text { Daily dose: }|\quad| \text { mg }\end{array}$ \\
\hline $\begin{array}{l}\text { Diuretics: Oral } \\
\text { (2nd medication) }\end{array}$ & $\begin{array}{l}\square \text { No } \\
\square \text { Bendrofluazide } \\
\square \text { Chlorthalidone } \\
\square \text { Hydrochlorothiazide } \\
\square \text { Furosemide } \\
\square \text { Indapamide } \\
\square \text { Torasemide } \\
\square \text { Bumetanide } \\
\square \text { Other } \\
\text { Daily dose: }|\quad| \text { mg }\end{array}$ & $\begin{array}{l}\square \text { No } \\
\square \text { Bendrofluazide } \\
\square \text { Chlorthalidone } \\
\text { Hydrochlorothiazide } \\
\square \text { Furosemide } \\
\square \text { Indapamide } \\
\square \text { Torasemide } \\
\text { Bumetanide } \\
\square \text { Other } \\
\text { Daily dose: }|\quad| \text { mg }\end{array}$ \\
\hline Ivabradine & $\begin{array}{l}\text { No } \square \text { Yes } \\
\text { Daily dose: }\left|\_\right| \text {mg }\end{array}$ & $\begin{array}{ll}\begin{array}{l}\text { No } \\
\text { If Yes, }\end{array} & \square \text { Yes } \\
\text { If No, } & \square \text { Daily dose: | } \mid \text { Atrial Fibrillation/Flutter } \\
& \square \text { EF }>35 \% \\
& \square \text { HR }<70 \text { bpm } \\
& \square \text { Medication still not available } \\
& \square \text { Other } \\
& \text { If Other: } \mid\end{array}$ \\
\hline Digitalis & $\square$ Yes & $\square$ Yes \\
\hline Statins & $\square$ No $\square$ Yes & $\square$ Yes \\
\hline Antiplatelets & $\square$ Yes & $\square$ Yes \\
\hline Anticoagulants & $\square$ Yes & $\square$ Yes \\
\hline Amiodarone & $\square$ Yes & $\square$ Yes \\
\hline Nitrates & $\square$ No $\quad \square$ Yes & $\square$ Yes \\
\hline $\begin{array}{l}\text { Calcium channel } \\
\text { blockers }\end{array}$ & $\square$ No $\square$ Yes & $\square$ Yes \\
\hline Antiarrhythmics & $\square$ Yes & $\square$ Yes \\
\hline $\begin{array}{l}\text { Direct renin } \\
\text { inhibitors }\end{array}$ & $\square$ No $\quad \square$ Yes & $\square$ Yes \\
\hline
\end{tabular}


Non CV drugs:

\begin{tabular}{|c|c|c|c|c|}
\hline Drug type & & Prior & \multicolumn{2}{|c|}{ During Outpatient Visi } \\
\hline Treatment for COPD & \multicolumn{2}{|c|}{$\begin{array}{l}\square \text { No } \\
\square \text { Corticosteroids } \\
\square \text { Beta2 agonists } \\
\square \text { Anticholinergic agents } \\
\square \text { Xanthine agents }\end{array}$} & \multicolumn{2}{|c|}{$\begin{array}{l}\square \text { No } \\
\square \text { Corticosteroids } \\
\square \text { Beta2 agonists } \\
\square \text { Anticholinergic agents } \\
\square \text { Xanthine agents }\end{array}$} \\
\hline Anti-diabetic drugs: Insulin & $\square$ No & $\square$ Yes & $\square$ No & $\square$ Yes \\
\hline Anti-diabetic drugs: Oral & \multicolumn{2}{|c|}{$\begin{array}{ll}\square & \text { Metformin } \\
\square & \text { Glitazones } \\
\square & \text { Incretins } \\
\square & \text { Sulphanylurea } \\
\square & \text { Other } \\
\square & \text { None }\end{array}$} & \multicolumn{2}{|c|}{$\begin{array}{l}\square \text { Metformin } \\
\square \text { Glitazones } \\
\square \text { Incretins } \\
\square \text { Sulphanylurea } \\
\square \text { Other } \\
\square \text { None }\end{array}$} \\
\hline Allopurinol & $\square$ No & $\square$ Yes & $\square$ No & $\square$ Yes \\
\hline NSAIDs: & $\square$ No & $\square$ Yes & $\square$ No & $\square$ Yes \\
\hline Antidepressants: & $\square$ No & $\square$ Yes & $\square$ No & $\square$ Yes \\
\hline Number of other non CV drugs: & \multicolumn{2}{|l|}{1} & \multicolumn{2}{|l|}{ L } \\
\hline
\end{tabular}




\section{Heart Failure Long-Term Registry \\ Medication: (Hospital Inpatients)}

\subsection{Medications and Doses}

\section{CV drugs:}

Doses should be total given in one day.

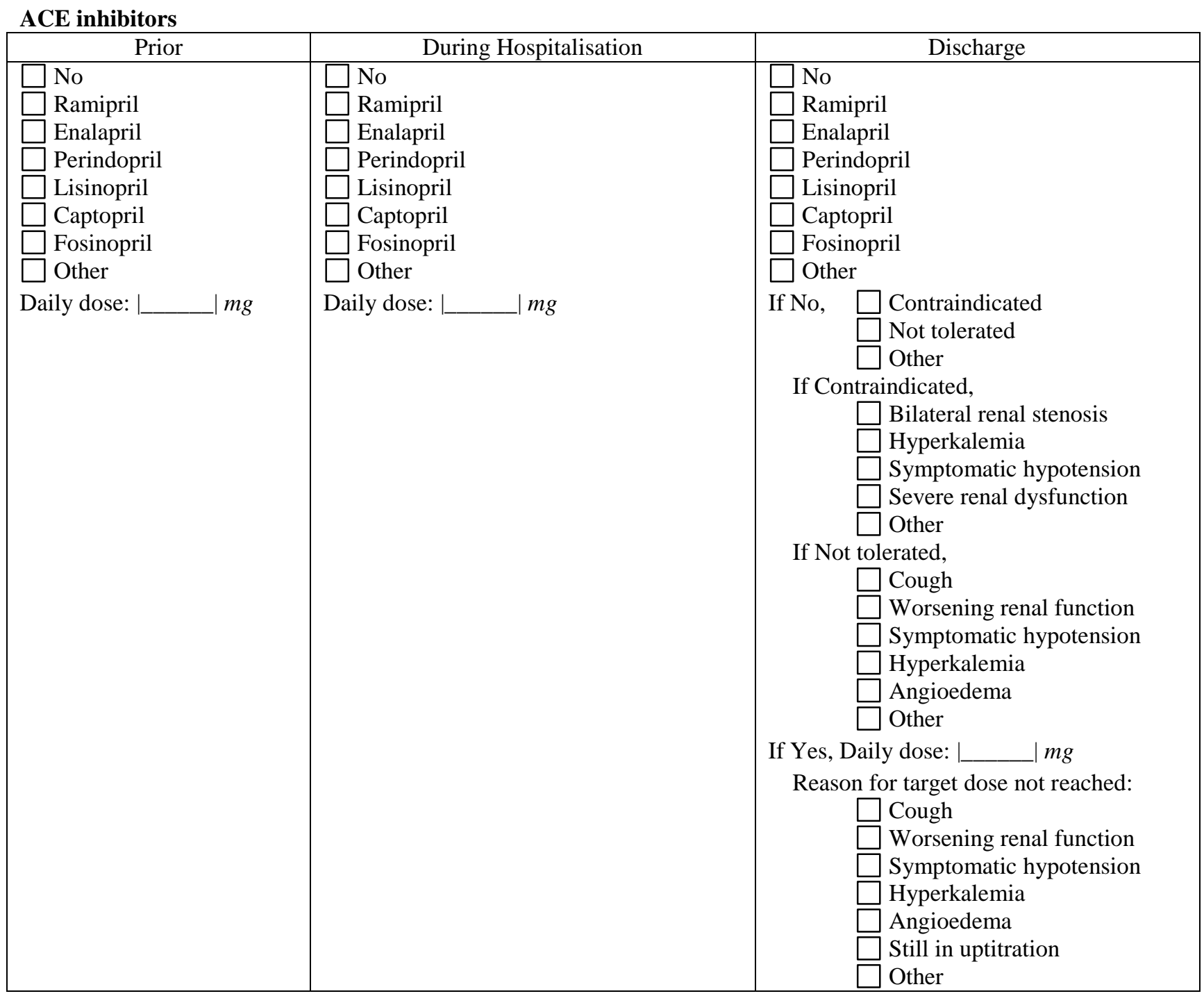


Angiotensin II Receptor Blockers (ARB)

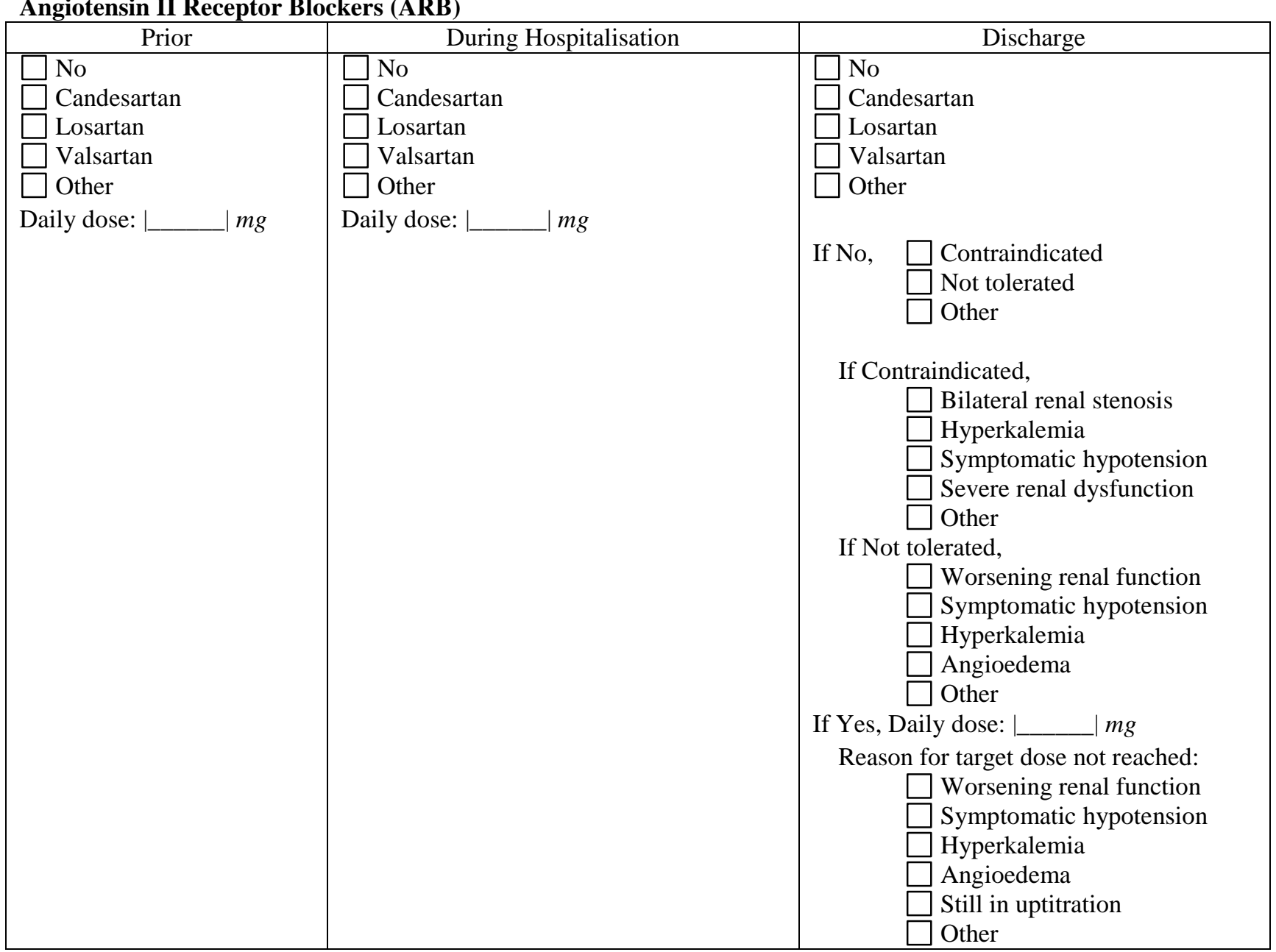

Sacubitril / Valsartan ARNI: Angiotensin Receptor Neprilysin Inhibitor)

\begin{tabular}{|c|c|c|}
\hline Prior & During Hospitalisation & Discharge \\
\hline$\square$ No & $\square$ No & $\square$ No \\
\hline$\square$ Yes & $\square$ Yes & $\square$ Yes \\
\hline Daily dose: $1 \ldots$ & Daily dose: $\perp_{\ldots} \mid \mathrm{mg}$ & \\
\hline & & $\begin{array}{l}\text { If No, } \square \text { Contraindicated } \square \text { Not } \\
\text { tolerated } \square \text { Other }\end{array}$ \\
\hline & & If Yes, Daily dose: $\left|\_\_\right| m g$ \\
\hline
\end{tabular}


Beta blockers

\begin{tabular}{|c|c|c|}
\hline Prior & During Hospitalisation & Discharge \\
\hline$\square$ No & $\square$ No & No \\
\hline$\square$ Carvedilol & $\square$ Carvedilol & $\square$ Carvedilol \\
\hline$\square$ Bisoprolol & $\square$ Bisoprolol & $\square$ Bisoprolol \\
\hline$\square$ Metoprolol & $\square$ Metoprolol & Metoprolol \\
\hline$\square$ Nebivolol & $\square$ Nebivolol & Nebivolol \\
\hline$\square$ Other & $\square$ Other & $\square$ Other \\
\hline Daily dose: $L_{\ldots} \mid m g$ & Daily dose: $|\ldots| m g$ & If No, $\square$ Contraindicated \\
\hline & & $\square$ Not tolerated \\
\hline & & $\square$ Other \\
\hline & & If Contraindicated, \\
\hline & & Asthma \\
\hline & & Bradyarrhythmia \\
\hline & & $\square$ PAD \\
\hline & & $\square$ Symptomatic hypotension \\
\hline & & $\square$ Other \\
\hline & & If Not tolerated, \\
\hline & & $\square$ Broncospasm \\
\hline & & Worsening PAD \\
\hline & & Worsening HF \\
\hline & & $\square$ Bradyarrhythmia \\
\hline & & Sexual dysfunction \\
\hline & & Symptomatic hypotension \\
\hline & & Other \\
\hline & & If Yes, Daily dose: $\left.\right|_{\perp} \mid m g$ \\
\hline & & Reason for target dose not reached: \\
\hline & & Broncospasm \\
\hline & & Worsening PAD \\
\hline & & Worsening HF \\
\hline & & $\square$ Bradyarrhythmia \\
\hline & & Sexual dysfunction \\
\hline & & Symptomatic hypotension \\
\hline & & $\square$ Still in uptitration \\
\hline & & Other \\
\hline
\end{tabular}




\section{Mineralocorticoid receptor antagonists}

\begin{tabular}{|c|c|c|}
\hline Prior & During Hospitalisation & Discharge \\
\hline$\square$ No & \multirow{5}{*}{$\begin{array}{l}\text { No } \\
\text { Spironolactone } \\
\text { Eplerenone } \\
\text { Canrenone } \\
\text { Other }\end{array}$} & $\square$ No \\
\hline$\square$ Spironolactone & & $\square$ Spironolactone \\
\hline$\square$ Eplerenone & & $\square$ Eplerenone \\
\hline$\square$ Canrenone & & $\square$ Canrenone \\
\hline$\square$ Other & & $\square$ Other \\
\hline Daily dose: $\mid \ldots$ & If No, $\square$ Contraindicated & If No, $\square$ Contraindicated \\
\hline & Not tolerated & Not tolerated \\
\hline & Other & $\nabla$ Other \\
\hline & If Contraindicated, & If Contraindicated, \\
\hline & $\square$ Hyperkalemia & $\square$ Hyperkalemia \\
\hline & Severe renal dysfunction & Severe renal dysfunction \\
\hline & $\square$ Other & $\square$ Other \\
\hline & If Not tolerated, & If Not tolerated, \\
\hline & $\square$ Hyperkalemia & $\square$ Hyperkalemia \\
\hline & $\square$ Worsening renal function & Worsening renal function \\
\hline & $\square$ Gynecomastie & $\square$ Gynecomastie \\
\hline & $\square$ Other & Other \\
\hline & If Yes, Daily dose: $\left|\_\_\right| m g$ & If Yes, Daily dose: $\mid \_\_m g$ \\
\hline & Reason for target dose not reached: & Reason for target dose not reached: \\
\hline & Hyperkalemia & Ц Hyperkalemia \\
\hline & $\square$ Worsening renal function & $\bigsqcup$ Worsening renal function \\
\hline & $\square$ Gynecomastie & $\square$ Gynecomastie \\
\hline & $\square$ Still in uptitration & $\square$ Still in uptitration \\
\hline & Other & Other \\
\hline
\end{tabular}

\section{Diuretics oral}

\begin{tabular}{|c|c|c|}
\hline Prior & During Hospitalisation & Discharge \\
\hline$\square \mathrm{No}$ & $\square \mathrm{No}$ & $\square$ No \\
\hline Bendrofluazide & $\square$ Bendrofluazide & $\square$ Bendrofluazide \\
\hline$\square$ Chlorthalidone & $\square$ Chlorthalidone & $\square$ Chlorthalidone \\
\hline$\square$ Hydrochlorotiazide & $\square$ Hydrochlorotiazide & $\square$ Hydrochlorotiazide \\
\hline$\square$ Furosemide & $\square$ Furosemide & $\square$ Furosemide \\
\hline$\square$ Indapamide & $\square$ Indapamide & $\square$ Indapamide \\
\hline$\square$ Torasemide & $\square$ Torasemide & $\square$ Torasemide \\
\hline$\square$ Bumetanide & $\square$ Bumetanide & $\square$ Bumetanide \\
\hline$\square$ Other & Other & \begin{tabular}{|l}
$\square$ \\
Other
\end{tabular} \\
\hline Daily dose: | & Daily dose: | & Daily dose: | \\
\hline
\end{tabular}

Diuretics oral ( $2^{\text {nd }}$ medication $)$

\begin{tabular}{|c|c|c|}
\hline Prior & During Hospitalisation & Discharge \\
\hline No & No & $\square \mathrm{No}$ \\
\hline$\square$ Bendrofluazide & $\square$ Bendrofluazide & $\square$ Bendrofluazide \\
\hline$\square$ Chlorthalidone & $\square$ Chlorthalidone & $\square$ Chlorthalidone \\
\hline$\square$ Hydrochlorotiazide & $\square$ Hydrochlorotiazide & $\square$ Hydrochlorotiazide \\
\hline$\square$ Furosemide & $\square$ Furosemide & $\square$ Furosemide \\
\hline$\square$ Indapamide & $\square$ Indapamide & $\square$ Indapamide \\
\hline$\square$ Torasemide & $\square$ Torasemide & $\square$ Torasemide \\
\hline$\square$ Bumetanide & $\square$ Bumetanide & $\square$ Bumetanide \\
\hline$\square$ Other & Other & Other \\
\hline Daily dose: $1 \ldots m g$ & Daily dose: & Daily dose: \\
\hline
\end{tabular}




\section{Ivabradine}

\begin{tabular}{|c|c|c|}
\hline Prior & During Hospitalisation & Discharge \\
\hline$\square$ No $\square$ Yes & $\square$ No $\square$ Yes & $\square$ No $\square$ Yes \\
\hline If Yes, Daily dose: $\left|\_\right|$ & If Yes, Daily dose: $\mid \_\_m g$ & If Yes, Daily dose: $\mid \_\_m g$ \\
\hline & If No, $\square$ Atrial Fibrillation/Flutter & If No, $\square$ Atrial Fibrillation/Flutter \\
\hline & $\mathrm{EF}>35 \%$ & $\mathrm{EF}>35 \%$ \\
\hline & $\mathrm{HR}<70 \mathrm{bpm}$ & $\square \mathrm{HR}<70 \mathrm{bpm}$ \\
\hline & Medic. still not available & Medic. still not available \\
\hline & Not tolerated & Not tolerated \\
\hline & Other & Other \\
\hline & If Other: | & If Other: | \\
\hline
\end{tabular}

\begin{tabular}{|l|lc|lc|lc|}
\hline \multicolumn{1}{|c|}{ Drug type } & \multicolumn{2}{c|}{ Prior } & During Hospitalisation & \multicolumn{2}{c|}{ Discharge } \\
\hline Digitalis & $\square$ No & $\square$ Yes & $\square$ No $\quad \square$ Yes & $\square$ No & $\square$ Yes \\
\hline Statins & $\square$ No & $\square$ Yes & $\square$ No & $\square$ Yes & $\square$ No & $\square$ Yes \\
\hline Antiplatelets & $\square$ No & $\square$ Yes & $\square$ No & $\square$ Yes & $\square$ No & $\square$ Yes \\
\hline Anticoagulants & $\square$ No & $\square$ Yes & $\square$ No & $\square$ Yes & $\square$ No & $\square$ Yes \\
\hline Amiodarone & $\square$ No & $\square$ Yes & $\square$ No & $\square$ Yes & $\square$ No & $\square$ Yes \\
\hline Nitrates & $\square$ No & $\square$ Yes & $\square$ No & $\square$ Yes & $\square$ No & $\square$ Yes \\
\hline Calcium channel blockers & $\square$ No & $\square$ Yes & $\square$ No & $\square$ Yes & $\square$ No & $\square$ Yes \\
\hline Antiarrhythmics & $\square$ No & $\square$ Yes & $\square$ No & $\square$ Yes & $\square$ No & $\square$ Yes \\
\hline Direct renin inhibitors & $\square$ No & $\square$ Yes & $\square$ No & $\square$ Yes & $\square$ No & $\square$ Yes \\
\hline
\end{tabular}

Non CV drugs:

\begin{tabular}{|c|c|c|c|}
\hline Drug type & Prior & During Hospitalisation & Discharge \\
\hline Treatment for COPD & $\begin{array}{l}\text { No } \\
\square \text { Corticosteroids } \\
\square \text { Beta2 agonists } \\
\square \text { Anticholinergic agents } \\
\square \text { Xanthine agents }\end{array}$ & $\begin{array}{l}\square \text { No } \\
\square \text { Corticosteroids } \\
\square \text { Beta2 agonists } \\
\square \text { Anticholinergic agents } \\
\square \text { Xanthine agents }\end{array}$ & $\begin{array}{l}\square \text { No } \\
\square \text { Corticosteroids } \\
\square \text { Beta2 agonists } \\
\square \text { Anticholinergic agents } \\
\square \text { Xanthine agents }\end{array}$ \\
\hline Anti-diabetic drugs: Insulin & $\square$ Yes & $\square$ Yes & $\square$ Yes \\
\hline Anti-diabetic drugs: Oral & $\begin{array}{ll}\square \text { Metformin } \\
\square \text { Glitazones } \\
\square \text { Incretins } \\
\square \text { Sulphanylurea } \\
\square \text { Other } \\
\square \text { None }\end{array}$ & $\begin{array}{ll}\square \text { Metformin } \\
\square \text { Glitazones } \\
\square \text { Incretins } \\
\square \text { Sulphanylurea } \\
\square \text { Other } \\
\square \text { None }\end{array}$ & $\begin{array}{ll}\square & \text { Metformin } \\
\square & \text { Glitazones } \\
\square & \text { Incretins } \\
\square & \text { Sulphanylurea } \\
\square & \text { Other } \\
\square & \text { None }\end{array}$ \\
\hline Allopurinol & $\square$ Yes & $\square$ Yes & $\square$ Yes \\
\hline NSAIDs: & $\square$ Yes & $\square$ Yes & $\square$ Yes \\
\hline Antidepressants: & $\square$ Yes & $\square$ Yes & $\square$ Yes \\
\hline Number of non CV drugs: & 1 & 1 & 1 \\
\hline
\end{tabular}




\section{Heart Failure Long-Term Registry Discharge (hospitalised patients)}

\subsection{Discharge/Outcome}

Vital status: $\square$ Alive $\square$ Dead $\quad$ Date of discharge/death: $\mid \_/ /$dd/mm/yyy

If Dead,

Causes of death: $\square$ Procedure related $\square$ Non procedure related $\quad \square$ Unknown
Causes of death: $\square$ Cardiac $\quad \square$ Vascular $\quad \square$ Non cardiovascular $\quad \square$ Unknown

If cardiac causes, $\quad$ Mode: $\square$ Sudden $\square$ Non sudden

Causes: $\square$ AMI $\square$ Heart Failure $\square$ Arrhythmia $\square$ Other

If other cardiac cause, please specify:

If vascular causes, $\quad$ Details: $\square$ Ischemic stroke

$\square$ Hemorrhagic stroke

Systemic hemorrhage

Peripheral embolism

Pulmonary embolism

Time in Intensive Cardiac Care Unit: | days

\subsection{Biometrics}

If vital status $=$ Alive,

Weight: $\mathrm{kg}$

Blood pressure (Systolic/Diastolic):

Heart rate:

beats/min

NYHA class:

NYHA I

NYHA II $\mathrm{mmHg}$

\subsection{Physical Signs}

\begin{tabular}{|ll|ll|}
\hline Pulmonary rales: & $\square$ No $\square$ Yes & S3 gallop: & $\square$ No $\square$ Yes \\
\hline JVP(>6cm): & $\square$ No $\square$ Yes & Peripheral hypoperfusion: & $\square$ No $\square$ Yes \\
\hline Pleural effusion: & $\square$ No $\square$ Yes & Cold: & $\square$ No $\square$ Yes \\
\hline Hepatomegaly: & $\square$ No $\square$ Yes & Mitral regurgitation: & $\square$ No $\square$ Yes \\
\hline Peripheral oedema: & $\square$ No $\square$ Yes & Aortic stenosis: & $\square$ No $\square$ Yes \\
\hline
\end{tabular}

\subsection{Investigations/Procedures during hospitalisation}

ECG:

Performed $\square$ Not performed

Rhythm:

Heart rate:

QRS-duration:

QT-duration:

LBBB:

LVH:

\begin{tabular}{|l|l|l|l|l}
$\square$ Sinus \\
$\square$ \\
$\square$ No \\
$\square$ No \\
$\square$ No \\
$\square$ Yes \\
$\square$ Yes
\end{tabular}

Pathological Q-wave: $\square$ No $\square$ Yes 
Chest X-ray: $\square$ Performed $\square$ Not performed

Normal? No $\square$ Yes

If No, please specify:

\begin{tabular}{|ll|ll|}
\hline Cardiac enlargement: & $\square$ No $\square$ Yes & Pulmonary congestion: & $\square$ No $\square$ Yes \\
\hline Alveolar oedema: & $\square$ No $\square$ Yes & Other abnormality: & $\square$ No $\square$ Yes \\
\hline
\end{tabular}

If Other, please describe:

Echo-Doppler: $\quad \square$ Performed $\square$ Not performed

EF:

LVEDD:

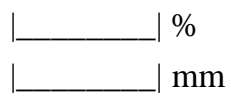

LVH:

No $\square$ Yes

E/A:

Deceleration time:

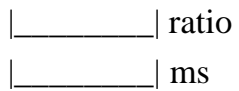

LA measurement:

$\square$ Volume $\square$ Dimension

IF Volume, LA Volume:

IF Dimension, LA Dimension:

Restrictive/pseudonormal pattern:

$\square$ No $\square$ Yes

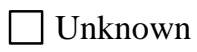

Mitral regurgitation moderate-severe:

$\square$ No $\square$ Yes

Aortic stenosis moderate-severe:

$\square$ No $\square$ Yes

Aortic regurgitation moderate-severe:

$\square$ No $\square$ Yes

Tricuspid regurgitation moderate-severe: $\square$ No $\square$ Yes

Exercise test: $\square$ No $\square$ Yes $\square$ Patient cannot do the test

Peak exercise, cycle ergometer:

Peak exercise, treadmill:

Peak VO2:

6 min walk test:

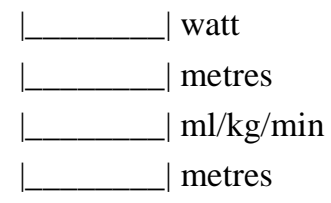

Holter Monitoring: $\quad \square$ Performed $\square$ Not performed

Mean HR:

PVC hour:

Unsustained VT:

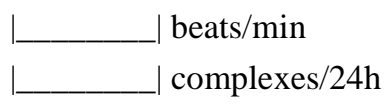

Sustained VT:

$\square$ No $\square$ Yes

Atrial fibrillation: $\quad \square$ No $\square$ Yes

$\square$ No $\square$ Yes

Coronary Angiography: $\square$ Performed $\square$ Not performed

Cardiac CT: $\quad \square$ Performed $\square$ Not performed

PCI/CABG: $\quad \square$ Performed $\square$ Not performed

EPS (Electrophysiological Study): $\quad \square$ Performed $\quad \square$ Not performed

Inducible Sustained VT/VF:

Inducible Atrial fibrillation:

Major conduction abnormalities:

$\square$ No $\square$ Yes
$\square$ No $\square$ Yes
$\square$ No $\square$ Yes


Transcatheter Ablation: Performed

Not performed

Atrial:

Ventricular:

Nodal:

$\begin{aligned} & \square \text { Performed } \square \text { Not performed } \\ & \square \text { No } \square \text { Yes } \\ & \square \text { No } \square \text { Yes } \\ & \square \text { No } \square \text { Yes }\end{aligned}$

Electric cardioversion:

$\square$ Performed $\square$ Not performed

Atrial Fibrillation

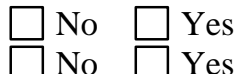

VT/VF:

No $\square$ Yes

Right Heart Catheterization:

mPAP:

Right atrial pressure:

PCW:

CI:

\begin{tabular}{|c|c|c|}
$\square$ Performed \\
$\mathrm{mmHg}$ \\
$\mathrm{mmHg}$ \\
$\mathrm{mmHg}$ \\
$\mathrm{L} / \mathrm{min} / \mathrm{m}^{2}$
\end{tabular}

Myocardial Scintigraphy:

$\square$ Performed $\square$ Not performed

Resting ischaemia:

$\square$ No $\square$ Yes

Myocardial viability:

No $\square$ Yes

Endomyocardial Biopsy: $\quad \square$ Performed $\quad \square$ Not performed

IAPB: $\quad \square$ Performed $\square$ Not performed

CRT implantation: $\quad \square$ Not indicated $\quad \square$ Indicated $\quad \square$ Already implanted

If Indicated, treatment:

$\square$ Not planned $\square$ Planned

If Not planned, reason:

$\square$ Absence of clinical indication
$\square$ Cost issues
$\square$ Patient refusal
$\square$ Logistic issues
$\square$ Other

ICD implantation:

$\square$ Not indicated $\square$ Indicated $\square$ Already implanted

If Indicated, treatment: Not planned $\square$ Planned

If Not planned, reason:

$\square$ Absence of clinical indication
$\square$ Cost issues
$\square$ Patient refusal
$\square$ Logistic issues
$\square$ Other

Education: $\quad \square$ No $\square$ Yes

If Yes, please specify:

\begin{tabular}{|ll|ll|}
\hline Heartfailurematters.org: & $\square$ No $\square$ Yes & Other web sites: & $\square$ No $\square$ Yes \\
\hline National booklets: & $\square$ No $\square$ Yes & Other: & $\square$ No $\square$ Yes \\
\hline
\end{tabular}

Rehabilitation: $\quad \square$ Performed $\square$ Not performed 


\subsection{Chemistry at Hospital Discharge / Outcome}

\begin{tabular}{|c|c|c|c|c|c|}
\hline White blood cells: & L & $\begin{array}{l}\square \text { Cells/microL } \\
\square \text { Giga/L }\end{array}$ & $\begin{array}{l}\text { Total } \\
\text { cholesterol: }\end{array}$ & L & $\begin{array}{l}\mathrm{mg} / \mathrm{dL} \\
\square \mathrm{mmol} / \mathrm{L}\end{array}$ \\
\hline Hemoglobin: & - & $\begin{array}{l}\square \mathrm{g} / \mathrm{dL} \\
\square \mathrm{mmol} / \mathrm{L} \\
\square \mathrm{g} / \mathrm{L}\end{array}$ & Fasting glucose: & L & $\begin{array}{l}\square \mathrm{mg} / \mathrm{dL} \\
\square \mathrm{mmol} / \mathrm{L}\end{array}$ \\
\hline S-creatinine: & $\mid$ & $\begin{array}{l}\square \mathrm{mg} / \mathrm{dL} \\
\square \mu \mathrm{mol} / \mathrm{L}\end{array}$ & HbA1c: & 1 & $\square \%$ \\
\hline Nitrogen measured by: & $\square \mathrm{BUN}$ & $\square$ Urea & BNP: & L & $\begin{array}{l}\square \mathrm{pg} / \mathrm{mL} \\
\square \mathrm{pmol} / \mathrm{L}\end{array}$ \\
\hline If $\mathrm{BUN}^{(9)}$ : & | & $\begin{array}{l}\square \mathrm{mg} / \mathrm{dL} \\
\square \mathrm{mmol} / \mathrm{L}\end{array}$ & NT-proBNP: & 1 & $\begin{array}{l}\square \mathrm{pg} / \mathrm{mL} \\
\square \mathrm{pmol} / \mathrm{L}\end{array}$ \\
\hline If urea: & | & $\begin{array}{l}\square \mathrm{mg} / \mathrm{dL} \\
\square \mathrm{mmol} / \mathrm{L}\end{array}$ & Sodium: & L_ & $\mathrm{mEq} / \mathrm{L}$ or $\mathrm{mmol} / \mathrm{L}$ \\
\hline Uric acid: & - & $\begin{array}{l}\square \mathrm{mg} / \mathrm{dL} \\
\square \mu \mathrm{mol} / \mathrm{L}\end{array}$ & Potassium: & - & $\mathrm{mEq} / \mathrm{L}$ or $\mathrm{mmol} / \mathrm{L}$ \\
\hline Proteinuria: & $\square$ No $\square$ & Yes & Bilirubin: & L & $\begin{array}{l}\square \mathrm{mg} / \mathrm{dL} \\
\square \mu \mathrm{mol} / \mathrm{L}\end{array}$ \\
\hline TSH: & | & $\mathrm{mIU} / \mathrm{L}$ & HIV infection: & $\square$ No $\square$ & Yes \\
\hline Troponin I or T: & | & $\mathrm{ng} / \mathrm{mL}$ or $\mathrm{pg} / \mathrm{mL}$ & Hs-CRP: & | & $\mathrm{mg} / \mathrm{L}$ \\
\hline Hs-Troponin I or T: & | & $\mathrm{ng} / \mathrm{mL}$ or $\mathrm{pg} / \mathrm{mL}$ & & & \\
\hline
\end{tabular}

\subsection{Scores}

Was prognosis evaluated using a risk score? $\square$ No $\square$ Yes

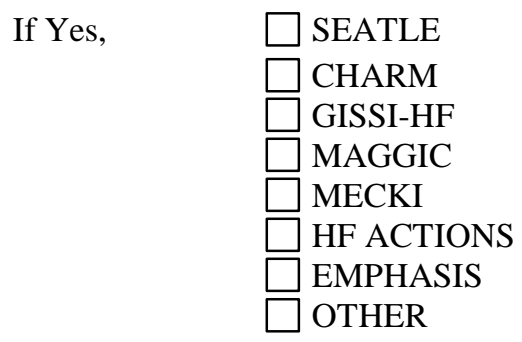

If Other, please specify:

References

- $\quad$ SEATLE : Wayne C; Levy et al - Circulation 2006, 113, 1424-1433

- CHARM : Stuart J. Pocock et al - European Heart Journal 2006, 27, 65-75

- GISSI-HF : Simona Barlera et al - Circulation Heart Failure 2013, 6, 31-39

- MAGGIC : Stuart J. Pocock et al - European Heart Journal 2012, 34, 1404-13

- $\quad$ MECKI : Piergiuseppe Agostini et al - Interventional Journal of Cardiology, 2012, 06-113

- HF ACTION: Christopher M. O’Connor et al - Circulation Heart Failure 2012, 5, 63-71

- $\quad$ EMPHASIS : Timothy J. Collier et al - European Heart Journal 2013, 34, 2823-9 


\section{Heart Failure Long-Term Registry Sign off}

\subsection{CRF Completed}

Answer YES to the question below to confirm that you have completed the questionnaire.

CRF Completed: $\quad \square$ No $\square$ Yes 


\section{Heart Failure Long-Term Registry 12-Month Follow-Up}

\subsection{Status at 12 months post inclusion}

Lost to follow-up: $\quad \square$ No $\square$ Yes If Yes, skip to section 7.4

Contact date: $\mid \_/ \_$___ dd/mm/yyyy $\quad \square$ Visit $\square$ Phone

Where is data collected: $\square$ Hospital $\square$ Primary care $\square$ HF clinic $\square$ Other organisation

By whom: $\quad \square$ Cardiologist $\square$ GP $\square$ Internal medicine doctor $\square$ Geriatrician $\square$ Nurse $\square$ Physiotherapist $\square$ Palliative care nurse $\quad \square$ Other

Days hospitalised prior year: | days

Vital status: $\square$ Alive $\square$ Dead

If Dead:

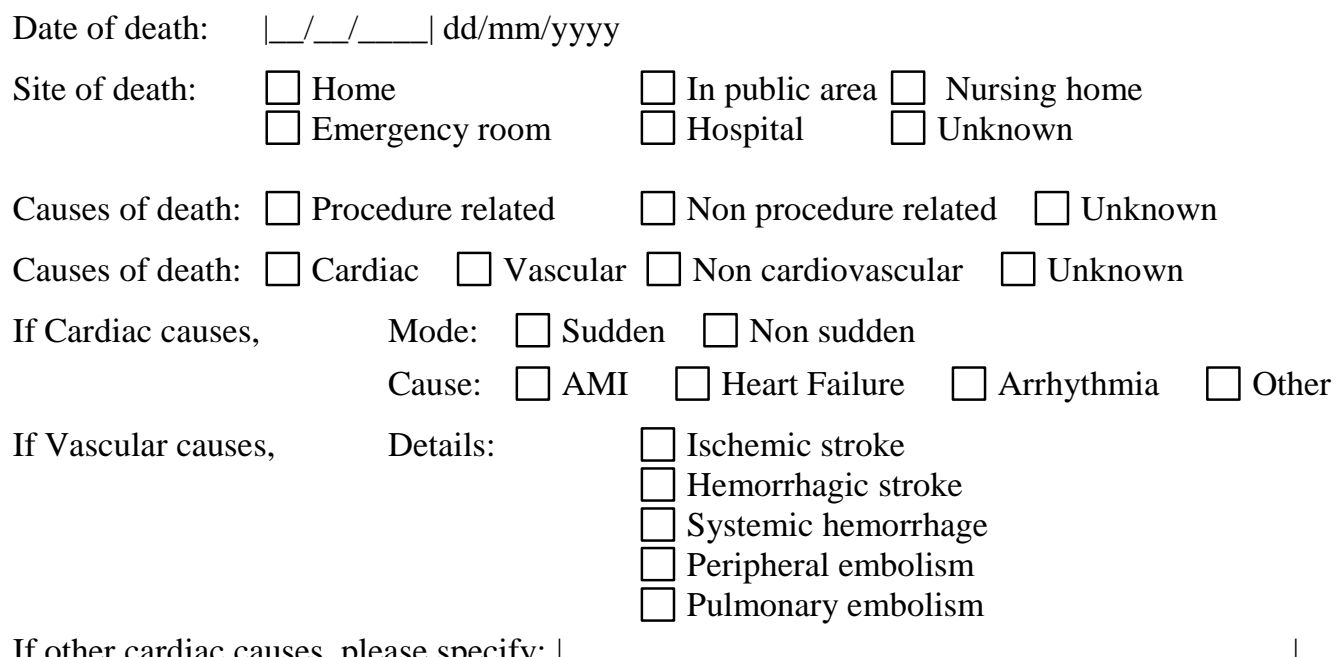

If Alive,

Heart rate: ___ _ _ _ _ beats/min

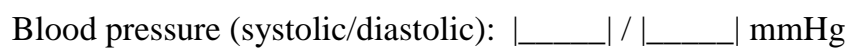

NYHA class: $\square$ NYHA I $\square$ NYHA II $\square$ NYHA III $\square$ NYHA IV

Re-hospitalisation (since discharge):

\begin{tabular}{|c|c|c|c|c|c|c|c|c|}
\hline \multirow{2}{*}{\multicolumn{2}{|c|}{ Re-hospitalisation }} & \multirow{2}{*}{$\begin{array}{c}\text { Date of } \\
\text { Re-hospitalisation } \\
\text { dd/mm/yyyy }\end{array}$} & \multirow{2}{*}{$\begin{array}{l}\text { Duration } \\
\text { (days) }\end{array}$} & \multicolumn{5}{|c|}{ Primary Cause } \\
\hline & & & & Cardiac, & $\mathrm{HF}$ & Vaccular & Renal & Non \\
\hline$\# 1$ & $\square$ No $\square$ Yes & 111 & & $\square$ & $\square$ & $\square$ & $\square$ & $\square$ \\
\hline$\# 2$ & $\square$ No $\square$ Yes & 111 & & $\square$ & $\square$ & $\square$ & $\square$ & $\square$ \\
\hline$\# 3$ & $\square$ No $\square$ Yes & 111 & & $\square$ & $\square$ & $\square$ & $\square$ & $\square$ \\
\hline \#4 & $\square$ No $\square$ Yes & 111 & & $\square$ & $\square$ & $\square$ & $\square$ & $\square$ \\
\hline$\# 5$ & $\square$ No $\square$ Yes & 111 & 1 & $\square$ & $\square$ & $\square$ & $\square$ & $\square$ \\
\hline
\end{tabular}




\subsection{Chemistry}

Blood test: $\square$ Performed $\square$ Not performed

\begin{tabular}{|c|c|c|c|c|c|}
\hline White blood cells: & L_ & $\begin{array}{l}\square \text { Cells/microL } \\
\square \text { Giga/L }\end{array}$ & $\begin{array}{l}\text { Total } \\
\text { cholesterol: }\end{array}$ & L & $\begin{array}{l}\square \mathrm{mg} / \mathrm{dL} \\
\square \mathrm{mmol} / \mathrm{L}\end{array}$ \\
\hline Hemoglobin: & | & $\begin{array}{l}\square \mathrm{g} / \mathrm{dL} \\
\square \mathrm{mmol} / \mathrm{L} \\
\square \mathrm{g} / \mathrm{L}\end{array}$ & Fasting glucose: & | & $\begin{array}{l}\square \mathrm{mg} / \mathrm{dL} \\
\square \mathrm{mmol} / \mathrm{L}\end{array}$ \\
\hline S-creatinine: & | & $\begin{array}{l}\square \mathrm{mg} / \mathrm{dL} \\
\square \mu \mathrm{mol} / \mathrm{L}\end{array}$ & HbA1c: & L & $\begin{array}{l}\% \\
\square \mathrm{mmol} / \mathrm{mol}\end{array}$ \\
\hline Nitrogen measured by: & $\square$ BUN & $\square$ Urea & BNP: & 1 & $\begin{array}{l}\square \mathrm{pg} / \mathrm{mL} \\
\square \mathrm{pmol} / \mathrm{L}\end{array}$ \\
\hline If $\mathrm{BUN}^{(9)}$ : & 1 & $\begin{array}{l}\square \mathrm{mg} / \mathrm{dL} \\
\square \mathrm{mmol} / \mathrm{L}\end{array}$ & NT-proBNP: & | & $\begin{array}{l}\square \mathrm{pg} / \mathrm{mL} \\
\square \mathrm{pmol} / \mathrm{L}\end{array}$ \\
\hline If urea: & | & $\begin{array}{l}\square \mathrm{mg} / \mathrm{dL} \\
\square \mathrm{mmol} / \mathrm{L}\end{array}$ & Sodium: & | & $\mathrm{mEq} / \mathrm{L}$ or $\mathrm{mmol} / \mathrm{L}$ \\
\hline Uric acid: & | & $\begin{array}{l}\square \mathrm{mg} / \mathrm{dL} \\
\square \mu \mathrm{mol} / \mathrm{L}\end{array}$ & Potassium: & | & $\mathrm{mEq} / \mathrm{L}$ or $\mathrm{mmol} / \mathrm{L}$ \\
\hline Proteinuria: & $\square$ No $\square$ & Yes & Bilirubin: & L_ & $\begin{array}{l}\square \mathrm{mg} / \mathrm{dL} \\
\square \mu \mathrm{mol} / \mathrm{L}\end{array}$ \\
\hline TSH: & 1 & $\mathrm{mIU} / \mathrm{L}$ & HIV infection: & $\square$ No $\square$ & Yes \\
\hline Troponin I or T: & | & $\mathrm{ng} / \mathrm{mL}$ or $\mathrm{pg} / \mathrm{mL}$ & Hs-CRP: & 1 & $\mathrm{mg} / \mathrm{L}$ \\
\hline Hs-Troponin I or T: & | & $\mathrm{ng} / \mathrm{mL}$ or $\mathrm{pg} / \mathrm{mL}$ & & & \\
\hline
\end{tabular}




\subsection{Medication (11)}

Is the patient under any of the medication below? $\quad \square$ No $\square$ Yes

CV Drugs

Doses should be total given in one day.

\begin{tabular}{|c|c|}
\hline Drug type & Generic name and daily Dose \\
\hline ACE inhibitors & 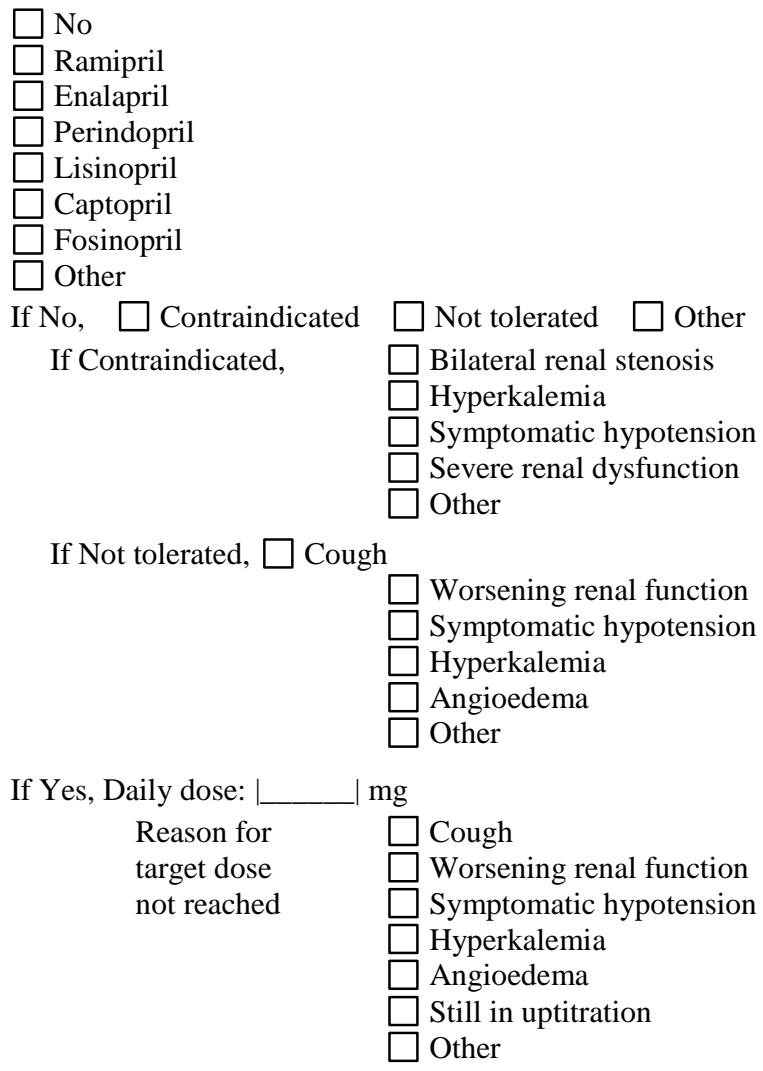 \\
\hline $\begin{array}{l}\text { Angiotensin II receptor } \\
\text { Blockers (ARB) }\end{array}$ & 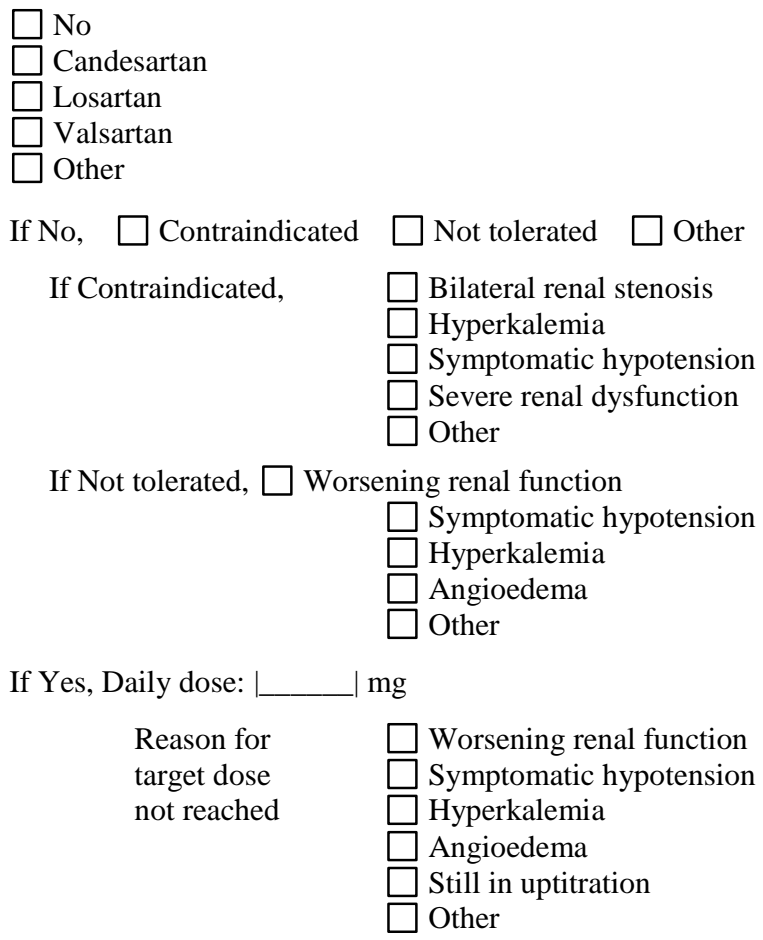 \\
\hline
\end{tabular}




\begin{tabular}{|c|c|}
\hline $\begin{array}{l}\text { Sacubitril / Valsartan } \\
\text { (ARNI: Angiotensin Receptor Neprilysin Inhibitor) }\end{array}$ & $\begin{array}{l}\square \text { No } \\
\square \text { Yes } \\
\text { If No, } \square \text { Contraindicated } \square \text { Not tolerated } \square \text { Other } \\
\text { If Yes, Daily dose: }\left|\_\right| \mathrm{mg}\end{array}$ \\
\hline Beta blockers & 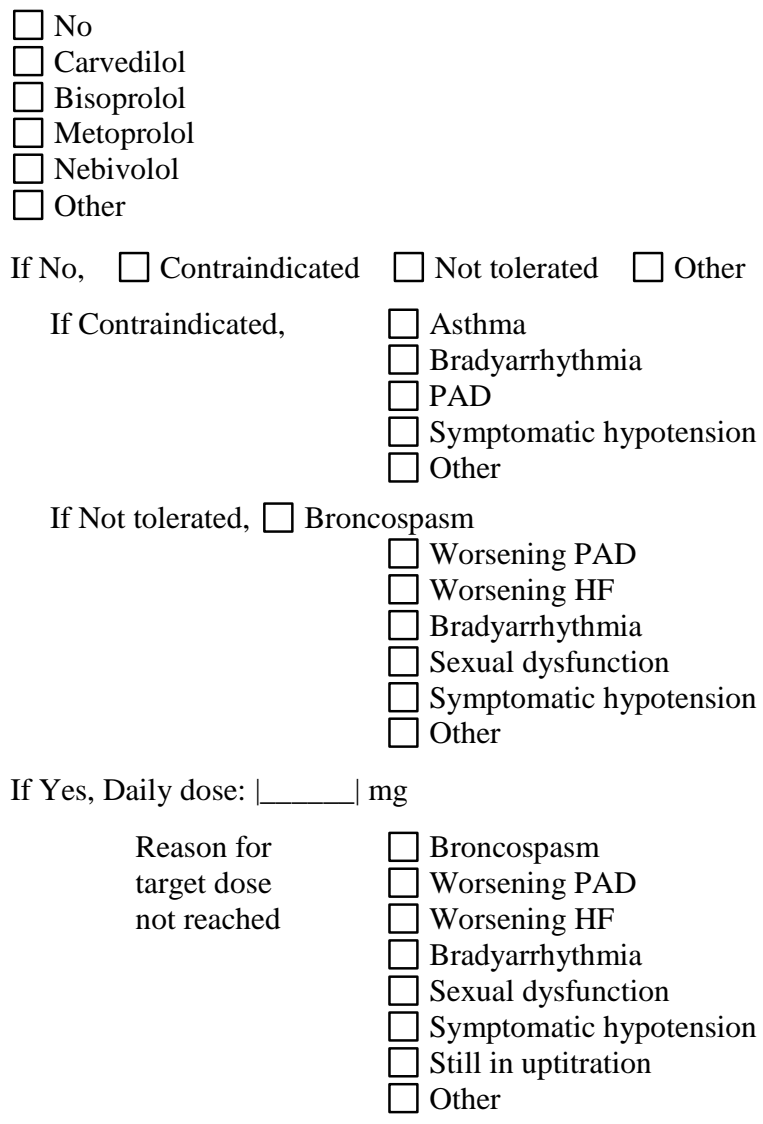 \\
\hline Mineralocorticoid receptor antagonists & 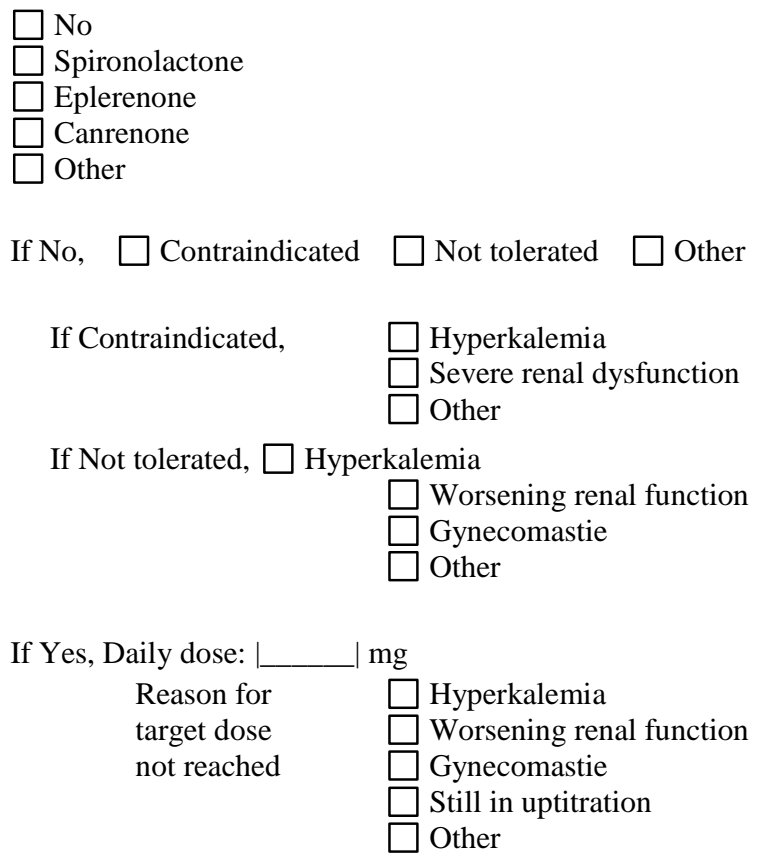 \\
\hline
\end{tabular}




\begin{tabular}{|c|c|}
\hline Diuretics: Oral & $\begin{array}{ll}\square \text { No } \\
\square \text { Bendrofluazide } \\
\square \text { Chlorthalidone } \\
\square \text { Hydrochlorothiazide } \\
\square \text { Furosemide } \\
\square \text { Indapamide } \\
\square \text { Torasemide } \\
\square \text { Bumetanide } \\
\square \text { Other } \quad \text { Daily dose: }|\quad| m g\end{array}$ \\
\hline Diuretics oral ( $2^{\text {nd }}$ medication $)$ & $\begin{array}{ll}\square \text { No } \\
\square \text { Bendrofluazide } \\
\square \text { Chlorthalidone } \\
\square \text { Hydrochlorothiazide } \\
\square \text { Furosemide } \\
\square \text { Indapamide } \\
\square \text { Torasemide } \\
\square \text { Bumetanide } \\
\square \text { Other }\end{array}$ \\
\hline Ivabradine & $\begin{aligned} \square \text { No } \quad \square \text { Yes } \quad \text { If Yes, Daily dose: }\left|\_\right| \text {mg } \\
\text { If No, } \square \text { Atrial Fibrillation/Flutter } \\
\square \mathrm{EF}>35 \% \\
\square \mathrm{HR}<70 \mathrm{bpm} \\
\square \text { Medication still not available } \\
\square \text { Not tolerated } \\
\square \text { Other If Other: } \mid\end{aligned}$ \\
\hline Digitalis & $\square$ Yes \\
\hline Statins & $\square$ No $\quad \square$ Yes \\
\hline Antiplatelets & $\square$ No $\quad \square$ Yes \\
\hline Anticoagulants & $\square$ No \\
\hline Amiodarone & $\square$ Yes \\
\hline Nitrates & $\square$ Yes \\
\hline Calcium channel blockers & $\square$ Yes \\
\hline Antiarrhythmics & $\square$ No $\quad \square$ Yes \\
\hline Direct renin inhibitors & $\square$ No $\square$ Yes \\
\hline
\end{tabular}

\section{Non CV drugs:}

\begin{tabular}{|c|c|}
\hline Treatment for COPD & $\begin{array}{l}\square \text { No } \\
\square \text { Corticosteroids } \\
\square \text { Beta2 agonists } \\
\square \text { Anticholinergic agents } \\
\square \text { Xanthine agents }\end{array}$ \\
\hline Anti-diabetic drugs: Oral & $\begin{array}{l}\square \text { Metformin } \\
\square \text { Glitazones } \\
\square \text { Incretins } \\
\square \text { Sulphanylurea } \\
\square \text { Other } \\
\square \text { None }\end{array}$ \\
\hline Anti-diabetic drugs: Insulin & $\square$ No $\quad \square$ Yes \\
\hline Allopurinol & $\square$ No $\square$ Yes \\
\hline NSAIDs: & $\square$ No $\quad \square$ Yes \\
\hline Antidepressants: & $\square$ No $\square$ Yes \\
\hline Number of non CV drugs: & 1 \\
\hline
\end{tabular}




\subsection{CRF Completed}

Answer YES to the question below to confirm that you have finished the 12 month follow-up data collection for this patient. Only completed CRFs will be taken into consideration for the analysis.

CRF Completed: $\square$ No $\square$ Yes 


\section{Help}

\begin{tabular}{|l|l}
\hline 1 & Outpatient $=$ All patients seen in the outpatient clinic.
\end{tabular}

Hospitalised $=$ All patients admitted for acute HF.

$2 \quad$ The date the patient was born as recorded on their birth certificate. Age should be greater than 18 years.

$3 \quad$ Ischaemic dilated cardiomyopathy should be classified as Ischaemic heart disease.

HFPEF Syndrome: one or more of hypertension, diabetes, obesity, older age, deconditioning, sleep apnea or others.

$4 \quad$ Patient currently enrolled in a randomised clinical trial? Will not affect enrolment as this is observational study.

$\begin{array}{ll}\text { 5 } & \text { Indicate if the patient has a history confirming any form of tobacco use in the past. This includes cigarettes, cigar }\end{array}$ and/or pipe.

Current $=$ patient regularly smokes a tobacco product / products one or more times per day or has smoked in the 30 days prior to this admission.

Former $=$ patient has stopped smoking tobacco products greater than 30 days before this admission .

$6 \quad$ Indicate if the patient has a history of hypertension diagnosed and/or treated by a physician

$\begin{array}{lll}6 & \text { Serum creatinine }>1.5 \mathrm{mg} / \mathrm{dL}\end{array}$

8 Viral, not alcoholic hepatitis

9 Blood urea nitrogen

$10 \quad$ Ventricular Tachycardia/Ventricular Fibrillation

\begin{tabular}{lll}
\hline 11 & Medications at hospital discharge/ambulatory visit
\end{tabular} 